\title{
Cell death: what can we learn from flies? Editorial for the special review issue on Drosophila apoptosis
}

\author{
Bertrand Mollereau
}

Published online: 24 July 2009

(C) Springer Science+Business Media, LLC 2009

Fifteen years after the identification of a small deletion (H99) in the Drosophila genome, revealing rpr, hid, and grim genes (RHG genes)[1], the field of apoptosis in Drosophila has grown dramatically. Models to study developmental apoptosis have diversified due to the powerful genetic tools in Drosophila and the numerous roles played by apoptosis in its development. Compared with C-elegans, where the role of apoptosis is mainly to eliminate unwanted cells, apoptosis in Drosophila is more diverse and complex resembling that in mammals. Apoptosis in Drosophila not only controls cell proliferation, elimination of damaged or developmentally confused cells but also organ size and the architecture of the tissues.

This issue of the Apoptosis journal includes ten review papers by experts in the field of Drosophila apoptosis. They provide an overview of apoptosis studies and alternative death pathways in Drosophila [2-11]. As the field gets larger, data on regulation mechanisms of the core apoptotic pathway, new models and concepts pave the way for studying of apoptosis. In an attempt to see where the field is heading and to identify the remaining key questions, I provide an overview of some of the recent studies described in the special issue and highlight new concepts. Unfortunately, due to topic overlap and space constraints, several topics did not get a dedicated review in this issue, and I apologize to those whose work was not mentioned. Several of these topics got excellent coverage in recent reviews: cell death in compensatory proliferation [12], cell competition [13], the role of the Hippo/Salvador pathway

B. Mollereau $(\square)$

LBMC, UMR5239 CNRS/Ecole Normale Supérieure de Lyon, IFR 128 Biosciences Lyon Gerland, Université de Lyon, Lyon, France

e-mail: bertrand.mollereau@ens-lyon.fr in apoptosis [14], the regulation of apoptosis in the pupal retina [15] and the role of alternative death pathways such as autophagy [16].

\section{Apoptosis during embryogenesis}

Zhou and Colleagues [2, this issue] focus on the historical model of apoptosis in Drosophila embryogenesis. The authors review the roles of apoptosis in development and its control mechanisms. During embryonic development, apoptosis eliminates supernumerary cells, controls cell proliferation and is also responsible for sculpting the tissue. The genes rpr, hid and grim are transcribed at about embryonic stage 11 and their products antagonize the Drosophila inhibitor of apoptosis, protein (DIAP1), removing the inhibition of caspases, and triggering apoptosis. It is still not fully understood how developmental pathways integrate both differentiation and apoptosis in tissues. The identification of developmental factors, such as Deformed, Abdominal B, which regulate RHG gene transcription [17], was a step towards the identification of $r p r$ transcriptional regulators but a thorough analysis of findings is needed to fully understand the transcriptional regulation of RHG genes. Recently, Zhou and colleagues showed that epigenetic regulations play an active part in the coordinated expression of $r p r$ and hid during apoptosis in embryos, suggesting that a complete understanding of RHG regulation may lie in alternative approaches [18].

In contrast to rpr and grim which are only expressed in cells destined to die, hid is also transcribed in some living cells. This suggests that in addition to transcriptional regulation, hid is also post-transcriptionally regulated. Bilak and $\mathrm{Su}$ cover in detail the mechanisms of Hid regulation [3, this issue] in developmental and DNA damage-induced 
apoptosis models. Hence, Hid can be regulated at the transcriptional, post-transcriptional and post-translational levels depending on the tissue or cell type in which it is expressed. Regulators include the Ras/MAPK, Hippo and JNK pathways, p53, the micro RNAs bantam, miR-6 and miR-2/13 and also the cell cycle regulators RBF1 and E2F.

\section{Inhibitor of apoptosis proteins}

Drosophila Inhibitor of apoptosis protein 1 (Diap1) is a critical inhibitor of apoptosis during Drosophila development. All living cells are thought to express Diap1, which restricts caspase activation. In embryos lacking Diapl, all cells enter apoptosis synchronously [19]. Orme and Meier review past and current findings on the complex interplay between the mechanisms regulating Diap1 and caspase protein stability [4, this issue].

IAP-mediated inhibition of caspase is a complex mechanism. Diap1 binding through its Baculovirus IAP repeat (BIR) domains to caspase is necessary, but not sufficient, for caspase inhibition. Diap1 also contains a really interesting new gene (RING) domain conferring E3 ubiquitin ligase activity, which transfers a ubiquitin group to caspases and supposedly targets them to the proteasome for degradation.

The story could have ended here but additional levels of complexity have recently been demonstrated. Firstly, Diapmediated caspase ubiquitylation causes inhibition by both degradative and non-degradative mechanisms. Diap1 mediates ubiquitylation of Dronc (caspase 9 homolog) but only targets it for degradation when it is associated with the Apaf-1-like protein, Dark, in the apoptosome [20]. However, Diap1-mediated ubiquitylation inhibits the effector caspase Drice (caspase 3 homolog) but does not induce its degradation. Instead the addition of ubiquitin groups to Drice sterically interferes with substrate binding and catalytic activity [21]. Secondly, these studies have shown that caspase-mediated cleavage of Diap1 [21] and Dark [20] is required to induce Diap1 inhibition. These auto-inhibitory feedback loops, may explain how caspase activation can be maintained at low levels in living cells and achieve a nonapoptotic function (see below "Roles of caspases in nonapoptotic cellular functions").

\section{The role of mitochondria in apoptosis}

The role of mitochondria in Drosophila is still the subject of numerous debates. In their review, Krieser and White present recent advances which have shed light on the role of members of the Bcl2 family and Cytochrome-C (Cyt-C). They also focus on the role of mitochondrial dynamics in apoptosis, a topic that has changed the debate [5, this issue].

Mitochondria have a central role in the intrinsic apoptotic pathway in vertebrates. Stimulation of apoptosis, causes members of the pro-apoptotic Bcl-2 family to trigger mitochondrial events, including the release of mitochondrial factors such as Cyt-C. Released Cyt-C interacts with Apaf-1 inducing the formation of the apoptosome, caspase activation and apoptosis (for review [22]).

It is believed that mitochondria only play a limited role in Drosophila apoptosis, in contrast to that in mammals [23]. This is because: (1) no developmental apoptosis defect was observed in mutants of the Drosophila bcl-2 homologs, debcl and buffy [24], 2) apoptosis occurs normally in Drosophila cell lines depleted in Cyt-C [25, 26] and 3) structural data showed that the apoptosome can form in the absence of Cyt-c in vitro [27]. Thus, it was suggested that the apoptosome is constitutively active and that the $\mathrm{rpr} / \mathrm{IAP}$ pathway is sufficient to explain apoptosis in Drosophila.

There is mounting evidence to challenge this view: (1) the Drosophila Bax/Bak ortholog debcl is required for some developmental apoptosis [28], (2) developmental apoptosis in the retina and non-apoptotic activation of caspase require Cyt-c [29-31] and (3) active apoptosome complexes are constantly subjected to feedback inhibition in living cells [20]. This suggests that activation of the apoptosome is not constitutive and that the apoptosome is activated by a signal (Cyt-c or another factor) in dying cells. Although the mechanisms by which Debcl and Cyt-c activate apoptosis remain unclear, these results suggest that mitochondria contribute to Drosophila apoptosis in several cellular contexts. The recent observation that mitochondrial fission is required for apoptosis, further implies that mitochondria have an important function in Drosophila apoptosis [32, 33].

\section{Cell death in germ cells and in the ovary}

$\mathrm{K}$. McCall and colleagues [6, this issue] give a complete and comprehensive overview of cell death models during oogenesis. Drosophila germ cells and ovaries offer a wide range of models for the study of developmental cell death, in addition to cell death brought about by nutrient deprivation, DNA damage, or environment-related risks such as cellular phone radiation [34, 35]. Cell death is normally observed during embryogenesis in germ cells although it also occurs in the germarium, and at mid- and late oogenesis in the adult female fly. However, with the exception of programmed cell death (PCD) in ovarian polar cells, PCD during oogenesis is not by classical apoptosis 
[36]. For example, none of the RHG genes is required for ovarian cell death. In addition, while the effector caspase $d c p-1$ has little or no role in most developmental apoptosis, it is required for autophagy and starvation-induced cell death in the germarium and at mid-oogenesis [37]. Mutations in autophagy genes reduced DNA fragmentation but not nuclear condensation. These results suggest a close relationship between apoptotic and autophagic pathways for ovarian cell death. The conditions and the molecular pathways by which a cell triggers apoptosis and/or autophagy need to be defined.

\section{Role of caspases in non-apoptotic cellular functions}

The mechanisms leading to caspase activation have been extensively studied during apoptosis. However, caspases can also be involved in vital cell processes. Arama and col. review the role of caspases during development in mammals and Drosophila models [7, this issue]. Apoptotic-like mechanisms in which part of the cell content is digested in a process that requires caspase activation are involved. This is, for example, the role of caspases during sperm terminal differentiation in Drosophila where the formation of the flagella requires the elimination of intra-cellular content [29]. Caspases are also involved in cellular compartment clearance during the process of dendritic pruning of sensory neurons [38].

Activated caspases also specifically regulate developmental pathways. For example, macrochaete differentiation is controlled by caspases. Apoptotic gene mutant flies exhibit one or several extra macrochaetes on their notum. One proposed mechanism is that the lack of caspase activation in sensory organ precursor (SOP) cells leads to Wingless signaling activation and the formation of an extra sensory neuron [39].

One of the mysteries in the field of apoptosis is how caspase activation can be restricted to particular developmental tasks without leading to cell death. There are several non-exclusive explanations: (1) This could be simply a question of levels, where low levels of caspase activation bring about subtle developmental regulation while high levels trigger apoptosis. Recent studies showing that caspases are activated at low levels in living cells to ensure their own inhibition [4, this issue] support this hypothesis. (2) A compartmentalized activation of caspases may ensure that only targeted processes are affected. (3) The production of apoptotic inhibitors may limit caspase activation to restricted areas or tasks. The identification factors controlling the spread of caspase activation within cells will be a major step forward in the understanding of apoptosis regulation.

\section{Endoplasmic reticulum stress and apoptosis}

There has been increasing interest in the contribution of the endoplasmic reticulum (ER), to the regulation of apoptosis. In some diseases such as diabetes and neurodegenerative diseases, misfolded/unfolded proteins accumulate in the ER, inducing ER stress and the activation of the unfolded protein response (UPR). Although sustained ER stress triggers apoptosis in certain cellular models (for review [40]), the role of ER stress in the progression of diseases remains unclear. Drosophila has only recently emerged as a biological model system to study ER stress and the UPR. Rasheva and Domingos [8, this issue] review the rapidly growing field of ER stress and apoptosis covering both the mammalian/yeast systems and the more recent studies using the Drosophila.

Studies on yeast and mammals have identified the UPR effectors that enable the cell to cope with the accumulation of unfolded/misfolded proteins in the ER. The UPR has primarily a protective role through the activation of Ire1/ Xpb1, Atf6 and Perk pathways. It results in the production of ER chaperones, activation of ER degradation proteins and translational attenuation, which lead to a reduction of misfolded protein load in the ER. However, in cells submitted to an intense or sustained ER stress, the UPR activates caspase-dependent cell death [40]. Caspases 9 and 3 are activated in ER stress-mediated apoptosis although their activation does not seem to require the mitochondrial Cyt-C/Apaf-1 pathway [41]. In mice, it has been proposed that calpains induce caspase 12 activation and subsequent activation of caspase 9 and 3 [42]. However, the role of caspase 12 in humans is not clear.

In a Drosophila autosomal dominant retinitis pigmentosa model, photoreceptor cells accumulate misfolded Rhodopsin 1 (Rh1) proteins that trigger ER stress. Here, the effectors of the Ire1/Xbp1 pathway limit the accumulation of misfolded Rh1 proteins and protect photoreceptor cells from death. In contrast, ectopic ER stress activation induced by expression of a spliced variant of $x b p 1$ leads to photoreceptor cell death that cannot be inhibited by the caspase inhibitor p35 [43]. This suggests that caspaseindependent death pathways may also be activated.

We have shown that induction of moderate ER stress in adult Drosophila photoreceptor cells does not trigger cell death but instead activates a protective mechanism that stimulates an anti-oxidant response and inhibits caspase activation [44]. Cultured mammalian cells subjected to mild ER stress also show a protective response that may be due to the instability of mRNAs and proteins that promote apoptosis [45]. Thus, the UPR not only limits the accumulation of misfolded proteins, but also protects tissues from apoptosis. It still has to be demonstrated in vivo whether moderate ER stress plays an anti-apoptotic role in 
normal and/or pathological conditions in mammals. Nevertheless, Drosophila promises to be a useful genetic model for the identification of apoptotic gene targets regulated by UPR effectors.

\section{Drosophila model of neurodegenerative diseases}

Human brain disorders, such as Alzheimer's, Parkinson's or Huntington's disease, are associated with neuronal cell loss but the pathways that lead to neurodegeneration are still under investigation. Apoptotic characteristics, such as caspase activation or TUNEL staining, are often detected in affected brain tissues but they play a limited role in neuron cell death. Neurodegenerative diseases commonly involve alternative death pathways induced by pathological proteins, which cause a disruption of mitochondrial dynamics and redox status. The situation is complex as many neuropathological models use distinct pathways to trigger cell death. B. W. Lu [9, this issue] exhaustively reviews the available Drosophila neurodegenerative models, ranging from Parkinson's and Alzheimer's to polyglutamine diseases. The number of Drosophila models has recently increased and they are now widely used for genetic and chemical compound screening.

The role of mitochondrial fission/fusion in neurological diseases is currently under deep scrutiny. Parkinson's disease models in Drosophila have shown that dynamin GTPases Drp1 and Opa1 regulate neurodegeneration [46-48]. Increased fusion or decreased fission may lead to neurodegeneration in pinkl and parkin mutants. Hence, enhanced fission by over production of Drp1 or reduced fusion by mutation in opal rescues dopaminergic neuron loss in pinkl mutant. This contrasts with the observation that Drp1-mediated fission is required for apoptosis (see the review on Mitochondrial apoptosis (in this issue [5]). One possible interpretation is that the role of fission in cell death may be context dependent. The exact relationship between mitochondrial dynamics and cell death needs to be determined. It is also unclear whether mechanisms identified in a particular neurodegenerative condition are applicable to other neurodegenerative diseases.

\section{JNK-dependent cell death}

In addition to the core apoptotic pathway, the c-Jun $\mathrm{N}$-terminal Kinase (JNK) pathway is also closely linked to cell death regulation. JNK is a pleiotropic pathway which regulates a broad range of biological processes, including proliferation, differentiation, morphogenesis, and apoptosis (for review see [49]). In Drosophila, JNK has been implicated in development in dorsal and thorax closure, imaginal disc eversion, planar cell polarity. It is also involved in the immune response, response to cellular damage, wound healing, longevity and apoptosis (for reviews covering these topics see [50-52]). T. Igaki gives an overview of the literature on JNK signaling and apoptosis in Drosophila [10, this issue].

Initial studies have shown that the activation of the Tumor Necrosis Factor/Tumor Necrosis Factor Receptor (TNF/TNFR) system in flies (Eiger/Wengen) induces JNKdependent cell death in stress situations but is not required for developmental apoptosis [53-55]. However, a recent study showed that JNK signaling contributed to morphogenetic apoptosis during normal development. Morphogenetic apoptosis is induced when a morphogenetic gradient is interrupted [56]. This allows a continuous gradient to be regenerated after a discontinuity. Until recently, this process was thought to be activated only in mutants (eg: mutant clone in morphogen signaling), but it is required for the normal joint formation in leg development. Hence, a sharp discontinuous Dpp signaling occurs during normal segmentation of the distal legs of Drosophila [57].

Recent work has demonstrated that JNK-mediated cell death suppresses pre-malignant cells via endocytic activation [58]. In this model, the Drosophila TNF-JNK signaling pathway is seen as tumor suppressor pathway. This study and previous work on JNK regulation illustrate what a powerful tool Drosophila genetics is in deciphering the multiple functions of pleiotropic pathways.

\section{Apoptotic corpse engulfment}

The removal of apoptotic cells by phagocytes is essential to avoid release of cellular contents and potential inflammatory response. Engulfment requires the expression of an "eat me" signal at the surface of the apoptotic cell, recognition by the engulfing cell, followed by internalization and processing of the dead cells. In their review Baker and col. summarize the recent findings on these pathways. They cover recognition, internalization, and processing mechanisms in Drosophila phagocytosis [11, this issue].

Regarding apoptosis, the popular view is that dying cells are recognized and engulfed by phagocytes. However, $C$-elegans studies show that engulfment genes take an active part in the activation of the apoptotic machinery. Hence, mutations in engulfment genes may enhance the survival of many cells normally destined to die [59, 60]. A recent study in Drosophila went further by showing that during the cell competition process, engulfing genes such as draper (drpr), myoblast city $(m b c)$ and Wiskott-Aldrich Syndrome (wasp) are required for the death of minutes cells (heterozygous mutants with a defective ribosomal protein) [61]. Baker and colleagues [11] discuss the evidence that 
may explain the mechanisms by which engulfing genes activate apoptosis in neighbouring cells.

\section{Conclusions}

Drosophila has proved to be a powerful tool for deciphering conserved regulatory mechanisms controlling cell death in living organisms. The comparison of cell death mechanisms between Drosophila and mammals has also revealed some differences. The study of these differences has enabled current views to be challenged and unexpected regulatory pathways in normal or pathological situations to be detected.

Acknowledgments BM projects are supported by French grants from the Fondation pour la Recherche Médicale (Equipment and Team programs) from the Centre National de la Recherche Scientifique (ATIP program), from the Ligue contre le cancer (Comités Savoie, Saône et Loire) and from the Région Rhone Alpes (HNV cluster).

\section{References}

1. White K et al (1994) Genetic control of programmed cell death in Drosophila. Science 264:677

2. Lin N, Zhang C, Pang J, Zhou L (2009) By design or by chance: cell death during Drosophila embryogenesis. Apoptosis (in this issue)

3. Bilak A, Su TT (2009) Regulation of Drosophila melanogaster pro-apoptotic gene hid. Apoptosis (in this issue)

4. Orme M, Meier P (2009) Inhibitor of Apoptosis Proteins Gatekeepers of Death. Apoptosis (in this issue)

5. Krieser RJ, White K (2009) Inside an enigma: do mitochondria contribute to cell death in Drosophila? Apoptosis (in this issue)

6. Pritchett TL, Tanner EA, McCall K (2009) Cracking open cell death in the Drosophila ovary. Apoptosis (in this issue)

7. Feinstein-Rotkopf Y, Arama E (2009) Can't live without them, can live with them: roles of caspases during vital cellular processes. Apoptosis (in this issue)

8. Rasheva VI, Domingos PM (2009) Regulation of the Unfolded Protein Response and Apoptosis. Apoptosis (in this issue)

9. Lu B (2009) Recent Advances in Using Drosophila to Model Neurodegenerative Diseases. Apoptosis (in this issue)

10. Igaki T (2009) Correcting developmental errors by apoptosis: lessons from Drosophila JNK signaling. Apoptosis (in this issue)

11. Fullard JF, Baker NE (2009) Clearance of apoptotic corpses. Apoptosis (in this issue)

12. Fan Y, Bergmann A (2008) Apoptosis-induced compensatory proliferation. The cell is dead. Long live the Cell!. Trends Cell Biol 18:467

13. Morata G, Martin FA (2007) Cell competition: the embrace of death. Dev Cell 13:1

14. Harvey K, Tapon N (2007) The Salvador-Warts-Hippo pathway-an emerging tumour-suppressor network. Nat Rev Cancer $7: 182$

15. Carthew RW (2007) Pattern formation in the Drosophila eye. Curr Opin Genet Dev 17:309
16. Neufeld TP, Baehrecke EH (2008) Eating on the fly: function and regulation of autophagy during cell growth, survival and death in Drosophila. Autophagy 4:557

17. Lohmann I, McGinnis N, Bodmer M, McGinnis W (2002) The Drosophila Hox gene deformed sculpts head morphology via direct regulation of the apoptosis activator reaper. Cell 110:457

18. Zhang $\mathrm{Y}$ et al (2008) Epigenetic blocking of an enhancer region controls irradiation-induced proapoptotic gene expression in Drosophila embryos. Dev Cell 14:481

19. Wang SL, Hawkins CJ, Yoo SJ, Muller HA, Hay BA (1999) The Drosophila caspase inhibitor DIAP1 is essential for cell survival and is negatively regulated by HID. Cell 98:453

20. Shapiro PJ, Hsu HH, Jung H, Robbins ES, Ryoo HD (2008) Regulation of the Drosophila apoptosome through feedback inhibition. Nat Cell Biol 10:1440

21. Ditzel $M$ et al (2008) Inactivation of effector caspases through nondegradative polyubiquitylation. Mol Cell 32:540

22. Jiang X, Wang X (2004) Cytochrome C-mediated apoptosis. Annu Rev Biochem 73:87

23. Oberst A, Bender C, Green DR (2008) Living with death: the evolution of the mitochondrial pathway of apoptosis in animals. Cell Death Differ 15:1139

24. Sevrioukov EA et al (2007) Drosophila Bcl-2 proteins participate in stress-induced apoptosis, but are not required for normal development. Genesis 45:184

25. Zimmermann KC, Ricci JE, Droin NM, Green DR (2002) The role of ARK in stress-induced apoptosis in Drosophila cells. J Cell Biol 156:1077

26. Dorstyn L, Mills K, Lazebnik Y, Kumar S (2004) The two cytochrome c species, DC3 and DC4, are not required for caspase activation and apoptosis in Drosophila cells. J Cell Biol 167:405

27. Yu X, Wang L, Acehan D, Wang X, Akey CW (2006) Threedimensional Structure of a Double Apoptosome Formed by the Drosophila Apaf-1 Related Killer. J Mol Biol 355:577

28. Galindo KA, Lu WJ, Park JH, Abrams JM (2009) The Bax/Bak ortholog in Drosophila, Debcl, exerts limited control over programmed cell death. Development 136:275

29. Arama E, Agapite J, Steller H (2003) Caspase activity and a specific cytochrome $\mathrm{C}$ are required for sperm differentiation in Drosophila. Dev Cell 4:687

30. Arama E, Bader M, Srivastava M, Bergmann A, Steller H (2006) The two Drosophila cytochrome C proteins can function in both respiration and caspase activation. EMBO J 25:232

31. Mendes CS et al (2006) Cytochrome c-d regulates developmental apoptosis in the Drosophila retina. EMBO Rep 7:933

32. Goyal G, Fell B, Sarin A, Youle RJ, Sriram V (2007) Role of mitochondrial remodeling in programmed cell death in Drosophila melanogaster. Dev Cell 12:807

33. Abdelwahid E et al (2007) Mitochondrial disruption in Drosophila apoptosis. Dev Cell 12:793

34. McCall K (2004) Eggs over easy: cell death in the Drosophila ovary. Dev Biol 274:3

35. Panagopoulos DJ, Chavdoula ED, Nezis IP, Margaritis LH (2007) Cell death induced by GSM $900-\mathrm{MHz}$ and DCS $1800-\mathrm{MHz}$ mobile telephony radiation. Mutat Res 626:69

36. Peterson JS et al (2007) Noncanonical cell death pathways act during Drosophila oogenesis. Genesis 45:396

37. Hou YC, Chittaranjan S, Barbosa SG, McCall K, Gorski SM (2008) Effector caspase Dcp-1 and IAP protein Bruce regulate starvation-induced autophagy during Drosophila melanogaster oogenesis. J Cell Biol 182:1127

38. Kuo CT, Zhu S, Younger S, Jan LY, Jan YN (2006) Identification of E2/E3 ubiquitinating enzymes and caspase activity regulating Drosophila sensory neuron dendrite pruning. Neuron 51:283 
39. Kanuka $\mathrm{H}$ et al (2005) Drosophila caspase transduces Shaggy/ GSK-3beta kinase activity in neural precursor development. EMBO J 24:3793

40. Szegezdi E, Logue SE, Gorman AM, Samali A (2006) Mediators of endoplasmic reticulum stress-induced apoptosis. EMBO Rep $7: 880$

41. Rao RV et al (2002) Coupling endoplasmic reticulum stress to the cell death program. An Apaf-1-independent intrinsic pathway. J Biol Chem 277:21836

42. Nakagawa $T$ et al (2000) Caspase-12 mediates endoplasmicreticulum-specific apoptosis and cytotoxicity by amyloid-beta. Nature 403:98

43. Ryoo HD, Domingos PM, Kang MJ, Steller H (2007) Unfolded protein response in a Drosophila model for retinal degeneration. EMBO J 26:242

44. Mendes CS et al (2009) ER stress protects from retinal degeneration. EMBO J 28:1296

45. Rutkowski DT et al (2006) Adaptation to ER stress is mediated by differential stabilities of pro-survival and pro-apoptotic mRNAs and proteins. PLoS Biol 4:e374

46. Deng H, Dodson MW, Huang H, Guo M (2008) The Parkinson's disease genes pink1 and parkin promote mitochondrial fission and/or inhibit fusion in Drosophila. Proc Natl Acad Sci U S A 105:14503

47. Poole AC et al (2008) The PINK1/Parkin pathway regulates mitochondrial morphology. Proc Natl Acad Sci U S A 105:1638

48. Yang $Y$ et al (2008) Pink1 regulates mitochondrial dynamics through interaction with the fission/fusion machinery. Proc Natl Acad Sci U S A 105:7070

49. Xia Y, Karin M (2004) The control of cell motility and epithelial morphogenesis by Jun kinases. Trends Cell Biol 14:94
50. Noselli S, Agnes F (1999) Roles of the JNK signaling pathway in Drosophila morphogenesis. Curr Opin Genet Dev 9:466

51. Kanda H, Miura M (2004) Regulatory roles of JNK in programmed cell death. J Biochem 136:1

52. Martin P, Parkhurst SM (2004) Parallels between tissue repair and embryo morphogenesis. Development 131:3021

53. Igaki $\mathrm{T}$ et al (2002) Eiger, a TNF superfamily ligand that triggers the Drosophila JNK pathway. EMBO J 21:3009

54. McEwen DG, Peifer M (2005) Puckered, a Drosophila MAPK phosphatase, ensures cell viability by antagonizing JNK-induced apoptosis. Development 132:3935

55. Luo X, Puig O, Hyun J, Bohmann D, Jasper H (2007) Foxo and Fos regulate the decision between cell death and survival in response to UV irradiation. EMBO J 26:380

56. Adachi-Yamada T, Fujimura-Kamada K, Nishida Y, Matsumoto K (1999) Distortion of proximodistal information causes JNKdependent apoptosis in Drosophila wing. Nature 400:166

57. Manjon C, Sanchez-Herrero E, Suzanne M (2007) Sharp boundaries of Dpp signalling trigger local cell death required for Drosophila leg morphogenesis. Nat Cell Biol 9:57

58. Igaki T, Pastor-Pareja JC, Aonuma H, Miura M, Xu T (2009) Intrinsic tumor suppression and epithelial maintenance by endocytic activation of Eiger/TNF signaling in Drosophila. Dev Cell $16: 458$

59. Hoeppner DJ, Hengartner MO, Schnabel R (2001) Engulfment genes cooperate with ced-3 to promote cell death in Caenorhabditis elegans. Nature 412:202

60. Reddien PW, Cameron S, Horvitz HR (2001) Phagocytosis promotes programmed cell death in C. elegans. Nature 412:198

61. Li W, Baker NE (2007) Engulfment is required for cell competition. Cell 129:1215 